\title{
NÍVEIS DE CONTAMINANTES INORGÂNICOS EM CACHAÇAS DA REGIÃO DO QUADRILÁTERO FERRÍFERO ARMAZENADAS EM COPOS IN NATURA DE ESTEATITO (PEDRA-SABÃO)
}

\author{
Isabela da Costa Fernandes, Maria Arlene Fausto, Olivia Maria de Paula Alves Bezerra e Kesia Diego Quintaes* \\ Escola de Nutrição, Universidade Federal de Ouro Preto, Campus Morro do Cruzeiro, S/N, 35400-000 Ouro Preto - MG, Brasil \\ Marcelo Antonio Morgano e Isabela Bissoli Cerqueira \\ Centro de Ciência e Qualidade de Alimentos, Instituto de Tecnologia de Alimentos, Av. Brasil, 2880, 13070178 Campinas - SP, Brasil
}

Recebido em 24/3/13; aceito em 8/7/13; publicado na web em 9/8/13

\begin{abstract}
LEVELS OF INORGANIC CONTAMINANTS IN BRAZILIAN CACHAÇAS FROM THE QUADRILÁTERO FERRÍFERO REGION STORED IN IN NATURA STEATITE (SOAPSTONE) CUPS. The levels of inorganic contaminants (As, $\mathrm{Cd}, \mathrm{Cu}, \mathrm{Ni}$ and $\mathrm{Pb}$ ) were measured in 18 Brazilian spirit beverages, before and after 4 contact cycles of $24 \mathrm{~h}$ each with in natura soapstone (steatite) cups. Results were compared to Brazilian regulation levels. Spirits contained As, $\mathrm{Cd}, \mathrm{Ni}$ and $\mathrm{Pb}$ within permitted levels. For $\mathrm{Cu}$, $5.6 \%$ of the brands were above the limit. The contact with soapstone cups decreased $\mathrm{Cu}$ levels in the spirits, while the other elements remained unchanged. The use of in natura soapstone cups was considered safe because this kind of vessel did not transfer inorganic contaminants to the spirits.
\end{abstract}

Keywords: cupper; alcohol; spirit drinks.

\section{INTRODUÇÃO}

Devido a sua abundância e propriedades o esteatito (pedra-sabão) tem sido usado pelo homem desde a Antiguidade. ${ }^{1,2}$ Em função da sua densidade, maciez e resistência ao calor a pedra-sabão é um material considerado ideal para produzir recipientes, panelas e utensílios pequenos. ${ }^{1,3-6}$ Embora a composição da pedra-sabão apresente variação geográfica, há certo perfil predominante, contendo talco e clorita como minerais majoritários. ${ }^{2,3,5-7}$ A resistência do esteatito ao tempo é determinada pelas condições ambientais somadas às petrográficas e petrofísicas. ${ }^{1,8}$ Todavia, condições ácidas são danosas à sua estrutura, tanto em laboratório ${ }^{8,9}$ como nas esculturas e edificações, ${ }^{1}$ podendo ser potencializadas por microrganismos..$^{10,11}$

Civilizações antigas impermeabilizavam com lipídios recipientes novos de pedra-sabão, processo denominado cura, reduzindo a porosidade e minimizando a perda de massa. ${ }^{2,49} \mathrm{~A}$ cura continua sendo aplicada às panelas de pedra-sabão, ${ }^{4,9,12}$ ao mesmo tempo que permite que elementos como $\mathrm{Ca}, \mathrm{Mg}$, Fe e Ni possam ser transferidos aos alimentos. ${ }^{2,9,12}$ Metais com potencial de toxicidade ao homem, como $\mathrm{Cu}, \mathrm{Cd}, \mathrm{Ni}, \mathrm{Pb}$ e As, podem estar presentes na composição do esteatito, ${ }^{7,9,13} \mathrm{e}$ a transferência desses metais durante armazenamento de líquidos em recipientes de pedra-sabão pode ocorrer, ainda que reduzida pela cura, podendo transferir quantidades tóxicas dos referidos elementos. ${ }^{9,14,15}$

Atualmente, são comercializados produtos de pedra-sabão que não são passíveis de cura, tais como jarros e copos, os quais têm indicação para uso com bebidas alcoólicas. Todavia, até o momento o comportamento da pedra-sabão não-curada exposta ao álcool etílico não foi avaliado. Adicionalmente, as bebidas alcoólicas comerciais podem ser veículos de elementos tóxicos, incluindo $\mathrm{Ni}$, em teor superior ao máximo aceitável. ${ }^{16}$ A cachaça, bebida destilada nacional produzida por fermentação da cana-de-açúcar, deve atender à legislação brasileira no que tange à presença dos contaminantes inorgânicos $\mathrm{Cu}, \mathrm{Pb}$ e As, limitados a 5,0; 0,2 e 0,1 mg/L, respectivamente, ${ }^{17}$ tendo sido constatado extrapolação nos teores destes elementos em cachaças vendidas em Araraquara (SP). ${ }^{18}$

\footnotetext{
*e-mail: kesiadq@gmail.com
}

Estudo com cachaça, terceira maior bebida alcoólica destilada mais comercializada no mundo, ${ }^{19}$ não constatou relação entre o perfil iônico e a procedência da bebida. ${ }^{20}$ Todavia, Fernandes et al (2005), analisando cachaças do Nordeste, Centro e Sul do Brasil, relataram que as nordestinas apresentavam maiores teores de $\mathrm{Pb}$ e $\mathrm{Cu}$ e, independentemente da procedência, todas necessitavam de melhoria no controle de qualidade quanto à contaminação por metais. ${ }^{21}$ Outros pesquisadores relataram que $25 \%$ das cachaças produzidas em alambiques no sul de Minas Gerais apresentavam teor de $\mathrm{Cu}$ acima do permitido pela legislação.22 Além dos equipamentos, a água utilizada na produção da bebida merece importância. Na região do Quadrilátero Ferrífero de Minas Gerais (QFMG) ${ }^{23}$ as águas apresentaram concentrações de As entre 2 a $2980 \mu \mathrm{g} / \mathrm{L}$, sugerindo a necessidade de um monitoramento periódico da água de abastecimento da região. ${ }^{24}$

Assim sendo, o presente estudo avaliou o teor de contaminantes inorgânicos (As, $\mathrm{Cd}, \mathrm{Cu}, \mathrm{Ni}$ e $\mathrm{Pb}$ ) transmitidos por copos novos de pedra-sabão in natura a cachaças do QFMG, mantidas em distintos tempos de contato com o material, tendo como parâmetro os níveis de contaminantes admitidos pela legislação brasileira. ${ }^{25}$

\section{PARTE EXPERIMENTAL}

Foram determinados os teores de $\mathrm{As}, \mathrm{Cd}, \mathrm{Pb}$ e $\mathrm{Ni}$ em dezoitos marcas de cachaças produzidas em Minas Gerais. Posteriormente, foi realizado um ensaio de migração de ciclo contínuo, sendo do tipo experimental fatorial com três fatores (teor alcoólico da cachaça, procedência do esteatito e tempo de armazenamento da cachaça nos copos de pedra-sabão in natura).

\section{Soluções}

Solução de álcool etílico a 43\% (v/v): álcool etílico (Merck, p.a.) diluído em água deionizada (18,2 $\mathrm{M} \Omega$ ).

Solução de ácido nítrico a 5\% (v/v): ácido nítrico 65\% (Merck, p.a.) diluído em água deionizada (18,2 $\mathrm{M} \Omega$ ).

A partir de soluções padrão de $1000 \mathrm{mg} / \mathrm{L}$ de As, Cd, Cu, Ni e $\mathrm{P}($ Merck, p.a.) foram construídas as curvas analíticas nos seguintes 
intervalos: de 0,005 a $1,0 \mathrm{mg} / \mathrm{L}$ para $\mathrm{As}, \mathrm{Cd}, \mathrm{Pb}$ e $\mathrm{Ni}$ e de 0,025 a $25 \mathrm{mg} / \mathrm{L}$ para o $\mathrm{Cu}$.

\section{Amostras}

Dezoito frascos de cachaças produzidas na região do QFMG e que se enquadravam na definição de cachaça adotada pelo Ministério de Agricultura, Pecuária e Abastecimento (MAPA) ${ }^{18}$ foram adquiridos, cada um de um fabricante distinto e com graduação alcoólica variando entre 40 a $46 \%$ (v/v), segundo a respectiva rotulagem. Detalhes sobre as cachaças estão descritos a seguir na Tabela 1.

Tabela 1. Teor alcoólico e procedência das cachaças analisadas no estudo

\begin{tabular}{ccc}
\hline $\begin{array}{c}\text { Identificação da } \\
\text { Cachaça }\end{array}$ & Teor Alcóolico (\%) & $\begin{array}{c}\text { Cidade de Procedência } \\
\text { (MG) }\end{array}$ \\
\hline A & 43,5 & Santa Bárbara \\
B & 41 & Itabirito \\
C & 43 & Brumadinho \\
D & 42 & Sabará \\
E & 44 & Belo Vale \\
F & 40 & Belo Horizonte \\
G & 46 & Ouro Preto \\
H & 45 & Congonhas \\
J & 40 & Betim \\
K & 40 & Itatiaiuçu \\
L & 41 & Itabirito \\
M & 41 & Itabirito \\
N & 42 & Jeceaba \\
O & 42 & São Joaquim de Bicas \\
P & 44 & Itatiaiuçu \\
Q & 44 & Congonhas \\
R & 45 & Congonhas \\
S & 45 & Belo Vale \\
\hline
\end{tabular}

\section{Materiais}

Copos novos de pedra-sabão in natura procedentes dos distritos rurais de Cachoeira do Brumado e de Santa Rita de Ouro Preto, ambos pertencentes ao QFMG, Brasil, foram adquiridos para estudo. Todos foram lavados com esponja polimérica macia nova e solução diluída de detergente comercial, com enxágue em água corrente seguido por água destilada. A secagem foi feita em estufa, a $150{ }^{\circ} \mathrm{C}$ por 10 minutos.

\section{Equipamento e condições de operação}

Foi utilizado um espectrômetro de emissão com fonte de plasma com acoplamento indutivo (ICP OES) (Vista MPX Varian, Mulgrave, Austrália), com visão axial, equipado com uma fonte de rádio frequência $(\mathrm{RF})$ de $40 \mathrm{MHz}$, um detector multielementar simultâneo de estado sólido do tipo CCD (Charge Coupled Device), uma bomba peristáltica, câmara de nebulização e um nebulizador sea spray. O sistema foi controlado pelo software ICP Expert e utilizou argônio líquido com pureza de 99,996 \% (Air Liquid, SP, Brasil) como gás de plasma. As condições experimentais otimizadas de operação do equipamento foram: potência de RF (1000 W); vazão do gás de nebulização $\left(0,9 \mathrm{~L} \mathrm{~min}^{-1}\right)$; vazão de argônio principal $\left(15 \mathrm{~L} \mathrm{~min}^{-1}\right)$; vazão de $\operatorname{argônio~auxiliar~}\left(1,5 \mathrm{~L} \mathrm{~min}^{-1}\right)$; correção de fundo (2 pontos); tempo de integração e de leitura (10 s); número de replicatas (3); configuração da tocha (axial) e comprimentos de onda, As (188,980 nm), Cd (214,439 nm), Cu (324,754 nm), Pb $(220,353 \mathrm{~nm})$ e $\mathrm{Ni}(221,648 \mathrm{~nm})$.

\section{Determinação dos contaminantes inorgânicos em cachaças}

Para a determinação de $\mathrm{Ni}, \mathrm{Cd}, \mathrm{Pb}, \mathrm{Cu}$ e $\mathrm{As}$ nas amostras de cachaça foram transferidos quantitativamente $5 \mathrm{~mL}$ das amostras de cachaça para tubos de vidro e seu volume foi reduzido a metade em bloco digestor a $95{ }^{\circ} \mathrm{C}$. Em seguida, foi adicionado $1,25 \mathrm{~mL}$ de ácido nítrico $65 \%$ e mantido em aquecimento a $95^{\circ} \mathrm{C}$ por $2 \mathrm{~h}$. Após resfriamento, essa solução foi transferida quantitativamente para balão volumétrico de $25 \mathrm{~mL}$ com água deionizada. As análises foram feitas em duplicata.

As provas em branco foram conduzidas em duplicata com béqueres de vidro. $\mathrm{O}$ volume utilizado foi de $14 \mathrm{~mL}$ de cachaça.

\section{Ensaio de migração}

Duas marcas de cachaça, as que apresentavam o menor (F) e o maior (G) teor alcoólico, 40 e $46 \%$ respectivamente, foram selecionadas para um ensaio de migração. A transferência de contaminantes foi avaliada em 4 ciclos de exposição contínua: 24, 48, 72 e 96 h. Para cada um dos ciclos e marca de bebida foram utilizados 6 copos ( 3 de cada procedência), totalizando 24 copos, 12 de cada procedência. No início do ciclo, cerca de $20 \mathrm{~mL}$ de cachaça foram adicionados ao copo, que foi tampado com vidro de relógio e mantido em temperatura ambiente sob luz artificial até finalizar o tempo do ciclo. Ao término de cada ciclo, a bebida foi transferida para um frasco âmbar novo e descontaminado, com fechamento hermético, devidamente identificado e mantido sob refrigeração a $3{ }^{\circ} \mathrm{C}$ até o momento de realização das análises, que foram feitas sequencialmente com reduzido tempo de contato entre os frascos e as amostras.

Também foram coletadas alíquotas de cada marca de cachaça e tratadas de maneira similar às demais amostras, sendo que o teor dos elementos investigados nestas foi considerado como sendo o tempo zero.

\section{Qualidade analítica}

A metodologia foi verificada seguindo as normas do Instituto Nacional de Metrologia, Normalização e Qualidade Industrial (INMETRO) ${ }^{26}$ sendo avaliados os parâmetros: linearidade, sensibilidade, seletividade, limite de detecção (LOD), exatidão, precisão. A precisão do método foi determinada pelo coeficiente de variação de sete repetições analíticas. A precisão intermediária foi realizada num intervalo de dois dias com o mesmo analista. A qualidade analítica foi verificada por ensaios de recuperação, com adição de solução padrão dos metais em meio aquoso de ácido nítrico a 5\%: baixa (0,05 mg/L), média ( $0,30 \mathrm{mg} / \mathrm{L})$ e alta $(0,75 \mathrm{mg} / \mathrm{L})$. Para o $\mathrm{Cu}$ foram usados os mesmos níveis de fortificação dos outros elementos e um nível mais alto $(5 \mathrm{mg} / \mathrm{L})$.

\section{Análise estatística}

Os valores médios dos elementos nas amostras de cachaça foram utilizados para os cálculos estatísticos, sendo deduzido o valor do ensaio branco de cada elemento. As análises estatísticas foram realizadas no software Stata para Mac Os (versão 11), ${ }^{27}$ sendo adotado 5\% de nível de significância. A normalidade de todos os dados foi verificada através do teste de Shapiro-Wilk e a homocedasticidade pelo teste de Levene.

As variáveis investigadas no ensaio de transferência foram: teor alcoólico, procedência dos copos e tempo de armazenamento nos copos. Para avaliar diferença no teor de contaminantes em cada cachaça, nos diferentes tempos, de acordo com a procedência dos copos de pedra-sabão, foi utilizada análise de variância ou o teste de Kruskall-Wallis, quando os dados apresentavam heterocedasticidade. 
Para avaliar diferença no teor de contaminantes em cada cachaça e por procedência de copo, de acordo com o tempo de armazenamento da bebida nos copos, foi utilizada a análise de variância seguida do teste de Bonferroni.

Para avaliar diferença entre o teor de contaminantes e as diferentes marcas de cachaça, foi utilizada análise de variância, seguida do teste de Bonferroni, para variáveis com distribuição normal; para os dados não-paramétricos foi utilizado o teste de Kruskall-Wallis. Para avaliar se o teor de cada um dos contaminantes sofreu influência da associação do tempo de armazenamento no copo de pedra-sabão, da procedência do copo e do teor alcoólico das amostras de cachaça, foi utilizada a ANOVA Three-Way. Após avaliar os efeitos principais, foi avaliada a presença de interações de segunda e de terceira ordem entre os fatores do modelo.

\section{RESULTADOS E DISCUSSÃO}

Os resultados obtidos na validação da metodologia analítica são apresentados na Tabela 2. A avaliação do desempenho da metodologia usada foi conduzida através de ensaios de recuperação em 3 níveis de fortificação de amostra com soluções padrões dos contaminantes adicionados no início dos ensaios. Os resultados obtidos variaram entre $97 \%$ e $118 \%$ para todos os contaminantes nos 3 níveis de fortificação estudados. A precisão da metodologia avaliada pelo coeficiente de variação de 7 repetições analíticas variou entre 1,0\% e 4,0\% para os elementos estudados e a precisão intermediária medida em dias diferentes de ensaio variou entre $2,6 \%$ e $7,0 \%$. Os limites de detecção foram obtidos a partir de 3 vezes o desvio padrão de 10 medidas de branco analítico. As curvas analíticas obtidas para os elementos foram lineares em toda a faixa de concentração estudada e os coeficientes de correlação foram superiores a 0,999.

A presença dos contaminantes inorgânicos $\mathrm{Cu}, \mathrm{Pb}$ e As nas bebidas alcoólicas teve como ponto de corte 5,0;0,2 e 0,1 mg/L, respectivamente, conforme prevê a legislação brasileira vigente. ${ }^{17}$ Para Ni e Cd, o limite máximo tolerável sendo de 3,0 e 0,2 mg/L, respectivamente..$^{25}$ As dezoito marcas de cachaça analisadas apresentaram teores médios de $\mathrm{As}, \mathrm{Cd}, \mathrm{Ni}$ e $\mathrm{Pb}$ em conformidade com a legislação brasileira. Com relação ao $\mathrm{Cu}$, apenas a marca $\mathrm{O}$ apresentou teores que extrapolaram os limites legais (Tabela 2).

Os teores de As variaram de 0,006 a $0,8 \mathrm{mg} / \mathrm{L}$, sendo distintos entre todas as marcas de cachaça $(\mathrm{p}<0,05)$. As marcas A, E, K, Q e $\mathrm{R}$ tiveram teores maiores de As do que as marcas B, C, F, G, H, J, $\mathrm{L}, \mathrm{M}, \mathrm{N}, \mathrm{P}$ e S. Os teores de Cd variaram de 0,002 a 0,004 mg/L, sendo que o teor de $\mathrm{Cd}$ das cachaças A e $\mathrm{G}$ foi significativamente maior quando comparado com as demais marcas. De forma similar ao As, o teor de $\mathrm{Cu}$ nas cachaças foi estatisticamente diferente entre todas as marcas, variando de 0,946 a $9,451 \mathrm{mg} / \mathrm{L}$. Quanto ao $\mathrm{Ni}$, os teores variaram de 0,002 a $0,007 \mathrm{mg} / \mathrm{L}$, sendo que as marcas $\mathrm{A}, \mathrm{B}$ e $\mathrm{C}$ apresentaram teores significativamente maiores de $\mathrm{Ni}$ (A, B: 0,014 e C: $0,020 \mathrm{mg} / \mathrm{L}$ ), quando comparadas com todas as demais. Não foram detectadas diferenças no teor de $\mathrm{Pb}$ entre as dezoito marcas de cachaça, que variou de 0,003 a 0,074 mg/L (Tabela 3).

As cachaças analisadas foram produzidas em conformidade com a legislação brasileira, uma vez que, das dezoito marcas estudadas, dezessete $(94,4 \%)$ estavam conformes para todos os contaminantes estudados. Esses resultados se contrapõem em parte ao encontrado por Nascimento et. al (1999) que estudaram o perfil mineral de 74 cachaças brasileiras, as quais se diferenciaram quanto ao teor de $\mathrm{Ni}$ e se assemelharam quanto ao teor de $\mathrm{Pb} .{ }^{20} \mathrm{Em}$ relação ao $\mathrm{Cu}$, que esteve acima do permitido pela legislação $(5 \mathrm{mg} / \mathrm{L})$ em $5,6 \%$ das marcas, outros estudos reportaram que 18,8 e $25 \%$ das cachaças mineiras apresentavam teores de $\mathrm{Cu}$ acima do limite legal. ${ }^{22,28} \mathrm{Considerando} \mathrm{que}$ a Agência Nacional de Vigilância Sanitária (ANVISA), ${ }^{29}$ em março de 2007, publicou resolução limitando o contato direto de cobre com alimentos e bebidas, sem especificar qual tipo de bebida, a redução no percentual de cachaças com elevado conteúdo de cobre pode representar uma adequação à norma, uma vez que a presença de cobre em cachaças tem sido atribuída em parte ao uso do alambique de cobre. ${ }^{30}$

O cobre é um componente da cachaça que advém do material usado no alambique na sua produção. Ao longo do processo de destilação e/ou do tempo em que o alambique está parado, há formação de carbonato básico de cobre (azinhavre), o qual pode ser dissolvido pelos vapores alcoólicos ácidos e transmitido à bebida de forma não intencional. A limpeza apropriada do alambique ou uso de filtro a base de resina de troca iônica ou de carvão ativado após a destilação são meios para obtenção de cachaça com teor aceitável de cobre. ${ }^{31}$

$\mathrm{Na}$ Tabela 4 são exibidos os valores dos contaminantes encontrados nas cachaças com maior e menor teor alcoólico expostas ao contato com copos de pedra-sabão de procedências distintas em tempo de até 96 horas.

Tabela 2. Resultados obtidos na validação da metodologia

\begin{tabular}{|c|c|c|c|c|c|c|}
\hline \multirow{2}{*}{ Elemento } & \multicolumn{3}{|c|}{ Exatidão } & \multirow{2}{*}{ Precisão (\%) } & \multirow{2}{*}{$\begin{array}{c}\text { Precisão Intermediária } \\
(\%)\end{array}$} & \multirow{2}{*}{$\mathrm{LOD}^{* *}\left(\mathrm{mg} \mathrm{L}^{-1}\right)$} \\
\hline & Nível $\left(\mathrm{mg} \mathrm{L}^{-1}\right)$ & Obtido $\left(\mathrm{mg} \mathrm{L}^{-1}\right)$ & $\operatorname{Rec}(\%)$ & & & \\
\hline \multirow{3}{*}{ As } & 0,05 & $0,049 \pm 0,002$ & 98 & \multirow{3}{*}{4} & \multirow{3}{*}{7} & \multirow{3}{*}{0,006} \\
\hline & 0,30 & $0,291 \pm 0,012$ & 97 & & & \\
\hline & 0,75 & $0,881 \pm 0,063$ & 117 & & & \\
\hline \multirow{3}{*}{$\mathrm{Cd}$} & 0,05 & $0,055 \pm 0,000$ & 110 & \multirow{3}{*}{1} & \multirow{3}{*}{3} & \multirow{3}{*}{0,002} \\
\hline & 0,30 & $0,299 \pm 0,004$ & 100 & & & \\
\hline & 0,75 & $0,793 \pm 0,031$ & 106 & & & \\
\hline \multirow{4}{*}{$\mathrm{Cu}$} & 0,05 & $0,056 \pm 0,001$ & 111 & \multirow{4}{*}{4} & \multirow{4}{*}{4} & \multirow{4}{*}{0,003} \\
\hline & 0,30 & $0,354 \pm 0,010$ & 118 & & & \\
\hline & 0,75 & $0,794 \pm 0,034$ & 106 & & & \\
\hline & 5,00 & $5,515 \pm 0,072$ & 110 & & & \\
\hline \multirow{3}{*}{$\mathrm{Ni}$} & 0,05 & $0,055 \pm 0,002$ & 111 & \multirow{3}{*}{1} & \multirow{3}{*}{4} & \multirow{3}{*}{0,002} \\
\hline & 0,30 & $0,310 \pm 0,004$ & 103 & & & \\
\hline & 0,75 & $0,790 \pm 0,034$ & 105 & & & \\
\hline \multirow{3}{*}{$\mathrm{Pb}$} & 0,05 & $0,054 \pm 0,004$ & 108 & \multirow{3}{*}{2} & \multirow{3}{*}{3} & \multirow{3}{*}{0,003} \\
\hline & 0,30 & $0,303 \pm 0,007$ & 101 & & & \\
\hline & 0,75 & $0,809 \pm 0,046$ & 108 & & & \\
\hline
\end{tabular}

\footnotetext{
* Rec= Recuperação; ** LOD=3s
} 
Tabela 3. Teores de As, Cd, Cu, Ni e Pb em cachaças produzidas no Quadrilátero Ferrífero de Minas Gerais (Brasil)

\begin{tabular}{|c|c|c|c|c|c|c|c|c|c|c|}
\hline \multirow{3}{*}{$\begin{array}{c}\begin{array}{c}\text { Marcas de } \\
\text { cachaça }\end{array} \\
\text { A }\end{array}$} & \multirow{2}{*}{\multicolumn{2}{|c|}{$\frac{\text { As }\left(\mathrm{mg} \mathrm{L}^{-1}\right)}{\text { Média } \pm \mathrm{DP}}$}} & \multirow{2}{*}{\multicolumn{2}{|c|}{$\begin{array}{l}\mathrm{Cd}\left(\mathrm{mg} \mathrm{L}^{-1}\right) \\
\text { Média } \pm \text { DP }\end{array}$}} & \multirow{2}{*}{\multicolumn{2}{|c|}{$\begin{array}{l}\mathrm{Cu}\left(\mathrm{mg} \mathrm{L}^{-1}\right) \\
\text { Média } \pm \text { DP }\end{array}$}} & \multirow{2}{*}{\multicolumn{2}{|c|}{$\begin{array}{l}\mathrm{Ni}\left(\mathrm{mg} \mathrm{L}^{-1}\right) \\
\text { Média } \pm \mathrm{DP}\end{array}$}} & \multirow{2}{*}{\multicolumn{2}{|c|}{$\frac{\mathrm{Pb}\left(\mathrm{mg} \mathrm{L}^{-1}\right)}{\text { Média } \pm \mathrm{DP}}$}} \\
\hline & & & & & & & & & & \\
\hline & $0,036^{\mathrm{ae}}$ & $\pm 0,001$ & $0,004^{\mathrm{b}}$ & $\pm 0,001$ & $1,309^{\mathrm{a}}$ & $\pm 0,001$ & $0,014^{\text {ad }}$ & $\pm 0,005$ & $0,011^{\mathrm{a}}$ & $\pm 0,001$ \\
\hline B & $0,011^{\text {bd }}$ & $\pm 0,000$ & $0,003^{\mathrm{ab}}$ & $\pm 0,000$ & $1,906^{\mathrm{b}}$ & $\pm 0,008$ & $0,014^{\mathrm{ab}}$ & $\pm 0,000$ & $0,006^{\mathrm{a}}$ & $\pm 0,000$ \\
\hline $\mathrm{C}$ & $0,011^{\text {bd }}$ & $\pm 0,000$ & $0,003^{\mathrm{ab}}$ & $\pm 0,000$ & $3,945^{\mathrm{c}}$ & $\pm 0,006$ & $0,020^{\mathrm{b}}$ & $\pm 0,003$ & $0,070^{\mathrm{a}}$ & $\pm 0,009$ \\
\hline $\mathrm{D}$ & $0,080^{c}$ & $\pm 0,000$ & $0,003^{\mathrm{ab}}$ & $\pm 0,000$ & $4,410^{\mathrm{d}}$ & $\pm 0,006$ & $0,007^{\mathrm{cd}}$ & $\pm 0,000$ & $0,040^{\mathrm{a}}$ & $\pm 0,001$ \\
\hline E & $0,030^{\text {abe }}$ & $\pm 0,004$ & $0,003^{\mathrm{ab}}$ & $\pm 0,000$ & $1,113^{\mathrm{e}}$ & $\pm 0,003$ & $0,010^{\text {ade }}$ & $\pm 0,001$ & $0,020^{\mathrm{a}}$ & $\pm 0,001$ \\
\hline F & $0,011^{\text {bd }}$ & $\pm 0,000$ & $0,003^{\mathrm{ab}}$ & $\pm 0,000$ & $4,444^{\mathrm{f}}$ & $\pm 0,005$ & $0,006^{\mathrm{cd}}$ & $\pm 0,001$ & $0,030^{\mathrm{a}}$ & $\pm 0,001$ \\
\hline G & $0,011^{\text {bd }}$ & $\pm 0,000$ & $0,004^{\mathrm{b}}$ & $\pm 0,001$ & $4,598^{\mathrm{g}}$ & $\pm 0,001$ & $0,008^{\mathrm{cd}}$ & $\pm 0,001$ & $0,006^{\mathrm{a}}$ & $\pm 0,000$ \\
\hline $\mathrm{H}$ & $0,011^{\text {bd }}$ & $\pm 0,000$ & $0,003^{\mathrm{ab}}$ & $\pm 0,000$ & $2,133^{\mathrm{h}}$ & $\pm 0,006$ & $0,003^{\mathrm{cd}}$ & $\pm 0,000$ & $0,006^{\mathrm{a}}$ & $\pm 0,000$ \\
\hline $\mathrm{J}$ & $0,006^{\mathrm{d}}$ & $\pm 0,000$ & $0,002^{\mathrm{a}}$ & $\pm 0,000$ & $4,505^{\mathrm{i}}$ & $\pm 0,002$ & $0,002^{\mathrm{c}}$ & $\pm 0,000$ & $0,010^{\mathrm{a}}$ & $\pm 0,000$ \\
\hline K & $0,030^{\text {ae }}$ & $\pm 0,001$ & $0,002^{\mathrm{a}}$ & $\pm 0,000$ & $2,935^{\mathrm{j}}$ & $\pm 0,011$ & $0,002^{\mathrm{c}}$ & $\pm 0,000$ & $0,020^{\mathrm{a}}$ & $\pm 0,011$ \\
\hline $\mathrm{L}$ & $0,006^{\mathrm{d}}$ & $\pm 0,000$ & $0,002^{\mathrm{a}}$ & $\pm 0,000$ & $1,566^{\mathrm{k}}$ & $\pm 0,003$ & $0,002^{\mathrm{c}}$ & $\pm 0,000$ & $0,005^{\mathrm{a}}$ & $\pm 0,001$ \\
\hline M & $0,006^{\mathrm{d}}$ & $\pm 0,000$ & $0,002^{\mathrm{a}}$ & $\pm 0,000$ & $1,264^{1}$ & $\pm 0,002$ & $0,002^{\mathrm{c}}$ & $\pm 0,000$ & $0,003^{\mathrm{a}}$ & $\pm 0,000$ \\
\hline $\mathrm{N}$ & $0,006^{\mathrm{d}}$ & $\pm 0,000$ & $0,002^{\mathrm{a}}$ & $\pm 0,000$ & $3,963^{\mathrm{m}}$ & $\pm 0,002$ & $0,002^{\mathrm{c}}$ & $\pm 0,000$ & $0,010^{\mathrm{a}}$ & $\pm 0,000$ \\
\hline $\mathrm{O}$ & $0,020^{\text {abde }}$ & $\pm 0,001$ & $0,002^{\mathrm{a}}$ & $\pm 0,000$ & $9,451^{\mathrm{n}}$ & $\pm 0,007$ & $0,007^{\mathrm{cd}}$ & $\pm 0,000$ & $0,010^{\mathrm{a}}$ & $\pm 0,001$ \\
\hline $\mathrm{P}$ & $0,006^{\mathrm{d}}$ & $\pm 0,000$ & $0,002^{\mathrm{a}}$ & $\pm 0,000$ & $2,195^{\circ}$ & $\pm 0,005$ & $0,005^{\mathrm{ce}}$ & $\pm 0,000$ & $0,010^{\mathrm{a}}$ & $\pm 0,000$ \\
\hline Q & $0,039^{\mathrm{e}}$ & $\pm 0,003$ & $0,002^{\mathrm{a}}$ & $\pm 0,000$ & $2,238^{p}$ & $\pm 0,002$ & $0,002^{c}$ & $\pm 0,000$ & $0,040^{\mathrm{a}}$ & $\pm 0,022$ \\
\hline $\mathrm{R}$ & $0,037^{\mathrm{e}}$ & $\pm 0,006$ & $0,002^{\mathrm{a}}$ & $\pm 0,000$ & $1,336^{9}$ & $\pm 0,000$ & $0,002^{\mathrm{c}}$ & $\pm 0,000$ & $0,020^{\mathrm{a}}$ & $\pm 0,000$ \\
\hline S & $0,020^{\text {bd }}$ & $\pm 0,005$ & $0,002^{\mathrm{a}}$ & $\pm 0,000$ & $0,946^{\mathrm{r}}$ & $\pm 0,003$ & $0,002^{\mathrm{c}}$ & $\pm 0,000$ & $0,010^{\mathrm{a}}$ & $\pm 0,004$ \\
\hline
\end{tabular}

Teste de Análise de Variância One Way foi realizado com as seguintes variáveis: teores de As, Cd, Cu e Ni. Teste de mediana Kruskall-Wallis, foi realizado com a variável: teor de $\mathrm{Pb}$. As letras sobrescritas diferentes representam diferença estatisticamente significativa $(\mathrm{p}<0,05)$.

Tabela 4. Teor de As, $\mathrm{Cd}, \mathrm{Cu}, \mathrm{Ni}$ e $\mathrm{Pb}$ (ppm) em cachaças com diferentes teores alcoólicos, segundo a procedência do copo de pedra-sabão e o tempo de armazenamento

\begin{tabular}{|c|c|c|c|c|c|c|c|}
\hline \multirow{3}{*}{$\begin{array}{l}\frac{0}{0} \\
\stackrel{0}{0} \\
\frac{0}{0} \\
\frac{1}{I I}\end{array}$} & \multirow{3}{*}{ 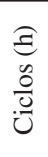 } & \multicolumn{2}{|c|}{ Cachaça F (grau alcoólico 40\%) } & \multirow{3}{*}{$\mathrm{P}$} & \multicolumn{2}{|c|}{ Cachaça G (grau alcoólico 46\%) } & \multirow{3}{*}{$\mathrm{P}$} \\
\hline & & $\begin{array}{c}\text { Copos procedentes de } \\
\text { Santa Rita } \\
\end{array}$ & $\begin{array}{l}\text { Copos procedentes de } \\
\text { Cachoeira do Brumado }\end{array}$ & & $\begin{array}{c}\text { Copos procedentes de } \\
\text { Santa Rita } \\
\end{array}$ & $\begin{array}{l}\text { Copos procedentes de } \\
\text { Cachoeira do Brumado }\end{array}$ & \\
\hline & & Média \pm DP & Média \pm DP & & Média \pm DP & Média \pm DP & \\
\hline \multirow{5}{*}{ As } & 0 & $0,010 \pm 0,001^{\mathrm{a}}$ & $0,006 \pm 0,001^{a}$ & 0,02 & $0,010 \pm 0,001^{\mathrm{a}}$ & $0,030 \pm 0,001^{\mathrm{a}}$ & 0,02 \\
\hline & 24 & $0,010 \pm 0,001^{\mathrm{b}}$ & $0,015 \pm 0,020^{\mathrm{b}}$ & 0,67 & $0,010 \pm 0,001^{\mathrm{a}}$ & $0,007 \pm 0,002^{\mathrm{b}, \mathrm{c}}$ & 0,01 \\
\hline & 48 & $0,070 \pm 0,070^{\mathrm{c}}$ & $0,008 \pm 0,004^{\mathrm{c}}$ & 0,20 & $0,040 \pm 0,040^{\mathrm{a}}$ & $0,050 \pm 0,021^{\mathrm{c}, \mathrm{b}}$ & 0,64 \\
\hline & 72 & $0,050 \pm 0,070^{\mathrm{d}}$ & $0,022 \pm 0,031^{\mathrm{d}}$ & 0,55 & $0,020 \pm 0,021^{\mathrm{a}}$ & $0,020 \pm 0,021^{\mathrm{d}, \mathrm{e}}$ & 0,84 \\
\hline & 96 & $0,080 \pm 0,050^{\mathrm{e}}$ & $0,006 \pm 0,001^{\mathrm{e}}$ & 0,06 & $0,080 \pm 0,121^{\mathrm{a}}$ & $0,006 \pm 0,002^{e, d}$ & 0,31 \\
\hline \multirow{5}{*}{$\mathrm{Cd}$} & 0 & $0,003 \pm 0,000^{\text {a }}$ & $0,002 \pm 0,000^{a}$ & 0,02 & $0,004 \pm 0,000^{\mathrm{a}}$ & $0,002 \pm 0,000^{a}$ & 0,02 \\
\hline & 24 & $0,003 \pm 0,000^{\mathrm{b}}$ & $0,002 \pm 0,000^{\mathrm{b}}$ & 0,02 & $0,003 \pm 0,000^{b}$ & $0,002 \pm 0,000^{\mathrm{b}}$ & 0,02 \\
\hline & 48 & $0,004 \pm 0,001^{\mathrm{c}}$ & $0,002 \pm 0,000^{\mathrm{c}}$ & 0,008 & $0,004 \pm 0,001^{\mathrm{c}}$ & $0,002 \pm 0,000^{\mathrm{c}}$ & 0,02 \\
\hline & 72 & $0,004 \pm 0,002^{\mathrm{d}}$ & $0,002 \pm 0,000^{\mathrm{d}}$ & 0,03 & $0,003 \pm 0,000^{\mathrm{d}}$ & $0,002 \pm 0,000^{\mathrm{d}}$ & 0,02 \\
\hline & 96 & $0,003 \pm 0,001^{\mathrm{e}}$ & $0,002 \pm 0,000^{\mathrm{e}}$ & 0,02 & $0,003 \pm 0,000^{\mathrm{e}}$ & $0,002 \pm 0,000^{\mathrm{e}}$ & 0,02 \\
\hline \multirow{5}{*}{$\mathrm{Cu}$} & 0 & $4,440 \pm 0,000^{\mathrm{a}}$ & $4,520 \pm 0,000^{a}$ & 0,02 & $4,600 \pm 0,000^{\mathrm{a}}$ & $4,220 \pm 0.001^{a}$ & 0,02 \\
\hline & 24 & $2,841 \pm 0,030^{\mathrm{a}, \mathrm{b}}$ & $3,430 \pm 0,990^{\mathrm{b}}$ & 0,36 & $3,901 \pm 0,081^{\mathrm{b}, \mathrm{a}}$ & $4,281 \pm 0.090^{b}$ & 0,006 \\
\hline & 48 & $2,790 \pm 0,090^{\mathrm{a}, \mathrm{c}}$ & $3,270 \pm 0,450^{\mathrm{c}}$ & 0,15 & $3,490 \pm 0,360^{c, a}$ & $4,210 \pm 0,130^{c}$ & 0,03 \\
\hline & 72 & $2,440 \pm 0,350^{\mathrm{a}, \mathrm{d}}$ & $2,640 \pm 1,040^{\mathrm{d}}$ & 0,78 & $3,501 \pm 0,160^{\mathrm{d}, \mathrm{a}}$ & $4,281 \pm 0,110^{d}$ & 0,002 \\
\hline & 96 & $2,180 \pm 0,170^{\text {a,b,c,e }}$ & $2,290 \pm 0,980^{\mathrm{e}}$ & 0,86 & $3,690 \pm 0,120^{\mathrm{e}}$ & $4,150 \pm 0,14^{\mathrm{e}}$ & 0,01 \\
\hline \multirow{5}{*}{$\mathrm{Ni}$} & 0 & $0,006 \pm 0,000^{\mathrm{a}}$ & $0,002 \pm 0,000^{\mathrm{a}}$ & 0,02 & $0,008 \pm 0,000^{\mathrm{a}}$ & $0,002 \pm 0,000^{\mathrm{a}}$ & 0,02 \\
\hline & 24 & $0,007 \pm 0,003^{\mathrm{b}}$ & $0,010 \pm 0,008^{b}$ & 0,48 & $0,040 \pm 0,0400^{\mathrm{b}}$ & $0,002 \pm 0,000^{\mathrm{b}}$ & 0,26 \\
\hline & 48 & $0,030 \pm 0,001^{\mathrm{c}}$ & $0,002 \pm 0,000^{\mathrm{c}}$ & 0,01 & $0,120 \pm 0,160^{c}$ & $0,020 \pm 0,020^{c}$ & 0,32 \\
\hline & 72 & $0,070 \pm 0,060^{\mathrm{d}}$ & $0,004 \pm 0,003^{\mathrm{d}}$ & 0,14 & $0,330 \pm 0,460^{\mathrm{d}}$ & $0,020 \pm 0,010^{\mathrm{d}}$ & 0,32 \\
\hline & 96 & $0,040 \pm 0,010^{\mathrm{e}}$ & $0,010 \pm 0,010^{\mathrm{e}}$ & 0,03 & $0,040 \pm 0,010^{\mathrm{e}}$ & $0,040 \pm 0,020^{\mathrm{e}}$ & 0,90 \\
\hline \multirow{5}{*}{$\mathrm{Pb}$} & 0 & $0,030 \pm 0,000^{\mathrm{a}}$ & $0,020 \pm 0,000^{\mathrm{a}}$ & 0,02 & $0,006 \pm 0,000^{\mathrm{a}}$ & $0,020 \pm 0,000^{\mathrm{a}}$ & 0,02 \\
\hline & 24 & $0,020 \pm 0,020^{\mathrm{b}}$ & $0,020 \pm 0,020^{\mathrm{b}}$ & 0,90 & $0,010 \pm 0,009^{\mathrm{b}}$ & $0,010 \pm 0,004^{\mathrm{b}}$ & 0,75 \\
\hline & 48 & $0,030 \pm 0,040^{c}$ & $0,009 \pm 0,010^{c}$ & 0,43 & $0,010 \pm 0,006^{\mathrm{c}}$ & $0,008 \pm 0,004^{\mathrm{c}}$ & 0,29 \\
\hline & 72 & $0,030 \pm 0,030^{\mathrm{d}}$ & $0,004 \pm 0,001^{\mathrm{d}}$ & 0,12 & $0,020 \pm 0,020^{\mathrm{d}}$ & $0,060 \pm 0,040^{\mathrm{d}}$ & 0,25 \\
\hline & 96 & $0,006 \pm 0,000^{\mathrm{e}}$ & $0,003 \pm 0,000^{\mathrm{e}}$ & 0,02 & $0,009 \pm 0,005^{\mathrm{e}}$ & $0,004 \pm 0,002^{\mathrm{c}}$ & 0,18 \\
\hline
\end{tabular}

Para um mesmo elemento, letras sobrescritas iguais na coluna indicam diferença estatisticamente significativa $(\mathrm{p}<0.05)$ determinada por ANOVA one-way, seguida do teste de Bonferroni. 
Houve um incremento no teor de As com o tempo de exposição da cachaça com menor teor alcoólico nos copos provenientes de Santa Rita, em contraposição ao comportamento da cachaça de maior teor alcoólico armazenada em copos da mesma procedência. O teor de Cd foi considerado estável, com mínima alteração em seu teor em função da graduação alcoólica da cachaça ou mesmo da procedência do copo de pedra-sabão.

Foi notada redução na quantidade de $\mathrm{Cu}$ presente na bebida de menor graduação alcoólica, independente da procedência do copo de pedra-sabão. No caso da cachaça com $46 \%$ de álcool, houve uma redução mais acentuada nas primeiras 48 horas de exposição da bebida nos copos de pedra-sabão de Santa Rita, enquanto que essa mesma cachaça, mantida em copos de pedra-sabão de Cachoeira do Brumado, apresentou oscilação inconstante no teor de $\mathrm{Cu}$ (Tabela 4).

$\mathrm{O}$ maior teor de $\mathrm{Cu}$ no início do ensaio em relação ao final indica que a pedra-sabão adsorveu o elemento nas primeiras 72 horas de contato, sendo isto possivelmente relacionado à composição da rocha que apresenta carbonatos de cálcio e elementos calcófilos. ${ }^{2,7,32,33} \mathrm{~A}$ constância no teor de $\mathrm{Cu}$ no último ciclo de contato $(96 \mathrm{~h})$ pode ser decorrente da saturação dos carbonatos presentes na superfície dos recipientes, limitando a adsorção de $\mathrm{Cu}$.

A quantidade de Ni observada na cachaça com maior graduação alcoólica mantida em copos de pedra-sabão provenientes de Santa Rita foi crescente até 72 horas de exposição, reduzindo seu teor com 96 horas de contato. Houve também redução na quantidade de $\mathrm{Pb}$ das cachaças mantidas em contato com copos de pedra-sabão in natura, independente da graduação alcoólica ou procedência dos copos (Tabela 4).

A seguir, a Tabela 5 apresenta os resultados da ANOVA ThreeWay comparando o teor dos elementos de interesse do estudo por teor alcoólico da cachaça, tempo de contato e procedência dos copos de pedra-sabão. Para o As não foram encontradas diferenças no teor entre as cachaças com diferentes graduações alcoólicas nem em relação ao tempo de contato com os copos de pedra-sabão. Após o ajuste pelo tempo de contato e pelo teor alcoólico foi observado que as cachaças armazenadas em copos provenientes de Cachoeira do Brumado apresentaram, em média, um teor de As $0,02 \mathrm{mg} / \mathrm{L}$ maior do que as cachaças armazenadas em copos da procedência Santa Rita $(p=0,04)$.

Para o Cd não foram observadas diferenças no teor deste elemento entre as cachaças e nem em relação ao tempo de armazenamento destas nos copos de pedra-sabão. Após o ajuste pelo tempo de armazenamento e pelo teor alcoólico foi observado que as cachaças mantidas em copos provenientes de Cachoeira do Brumado apresentaram, em média, um teor de $\mathrm{Cd}$ 0,001 mg/L maior do que as cachaças colocadas em copos da procedência Santa Rita $(\mathrm{p}<0,0005)$.

Com relação ao $\mathrm{Cu}$ foi notado que as cachaças mantidas em copos provenientes de Cachoeira do Brumado apresentaram, em média, um teor de $\mathrm{Cu}$ significativamente menor do que as cachaças colocadas em copos oriundos de Santa Rita, após o modelo ser ajustado pelo tempo de armazenamento e pelo teor alcoólico ( $\mathrm{p}=0,003)$. Foi encontrada interação entre as variáveis tempo e teor alcoólico, sendo que a cachaça com menor teor alcoólico apresentou valores médios de $\mathrm{Cu}$ significativamente menores do que a cachaça com maior graduação alcoólica em todos os tempos de armazenamento (24, 48, 72 e 96 h) e também menores do que o apresentado no tempo inicial, com diferença negativa média de 0,$96 ; 0,82 ; 1,34$ e 1,68 mg/L e de 1,34; 1,$45 ; 1,94$ e 2,25 mg/L em relação à cachaça com maior graduação alcoólica e valor inicial, respectivamente (Tabela 5).

Não foram encontradas diferenças estatisticamente significativas no

Tabela 5. Resultado da ANOVA Three-Way comparando o teor dos elementos (As, $\mathrm{Cd}, \mathrm{Cu}, \mathrm{Ni}$ e $\mathrm{Pb}$ ) por teor alcoólico da cachaça, tempo de contato e procedência dos copos de pedra-sabão

\begin{tabular}{|c|c|c|c|c|c|c|}
\hline Elementos & Efeito principal & SS & $\mathrm{Df}$ & MS & $\mathrm{F}(\mathrm{df})^{\mathrm{a}}$ & $P$ \\
\hline \multirow{5}{*}{ As } & Procedência & 0,006 & 1 & 0,006 & 4,39 & 0,04 \\
\hline & Tempo & 0,01 & 4 & 0,002 & 1,74 & 0,15 \\
\hline & Teor Alcoólico & $8,81 * 10^{-6}$ & 1 & $8,81 * 10^{-6}$ & 0,01 & 0,94 \\
\hline & Resíduos & 0,08 & 53 & 0,0015 & & \\
\hline & Total & 0,095 & 59 & 0,0016 & & \\
\hline \multirow{5}{*}{$\mathrm{Cd}$} & Procedência & 0,00003 & 1 & 0,00003 & 110,95 & $<0,00005$ \\
\hline & Tempo & $1,57 * 10^{-6}$ & 1 & $3,92 * 10^{-7}$ & 1,55 & 0,20 \\
\hline & Teor Alcoólico & $1,67 * 10^{-8}$ & 1 & $1,67 * 10^{-8}$ & 0,07 & 0,80 \\
\hline & $\underline{\text { Resíduos }}$ & 0,00001 & 53 & $2,52 * 10^{-7}$ & 53 & \\
\hline & Total & 0,00004 & 59 & $7,28 * 10^{-7}$ & 59 & \\
\hline \multirow{6}{*}{$\mathrm{Cu}$} & Procedência & 1,72 & 1 & 1,72 & 9,5 & 0,003 \\
\hline & Tempo & 13,8 & 4 & 3,44 & 19 & $<0,00005$ \\
\hline & Teor Alcoólico & 13,44 & 1 & 13,44 & 74,11 & $<0,00005$ \\
\hline & Teor Alcoólico x Tempo & 5,3 & 4 & 1,32 & 7,29 & 0,0001 \\
\hline & $\underline{\text { Resíduos }}$ & 8,9 & 49 & 0,18 & & \\
\hline & Total & 43,13 & & 0,73 & 59 & \\
\hline \multirow{6}{*}{$\mathrm{Ni}$} & Procedência & 0,05 & 1 & 0,05 & 4,00 & 0,05 \\
\hline & Tempo & 0,08 & 4 & 0,02 & 1,57 & 0,20 \\
\hline & Teor Alcoólico & 0,03 & 1 & 0,03 & 2,57 & 0,11 \\
\hline & Resíduos & 0,64 & 53 & 0,012 & 53 & \\
\hline & $\underline{\text { Total }}$ & 0,8 & & 0,013 & 59 & \\
\hline & Tempo & 0,003 & 4 & 0,0008 & 2,56 & 0,05 \\
\hline \multirow{5}{*}{$\mathrm{Pb}$} & Procedência & 0,00002 & 1 & 0,00002 & 0,08 & 0,77 \\
\hline & Teor Alcoólico & 0,00009 & 1 & 0,00009 & 0,28 & 0,60 \\
\hline & Procedência x Teor Alcoólico & 0,002 & 1 & 0,002 & 4,93 & 0,03 \\
\hline & $\underline{\text { Resíduos }}$ & 0,02 & 52 & 0,0003 & & \\
\hline & Total & 0,02 & 59 & 0,0003 & 59 & \\
\hline
\end{tabular}


teor de Ni das cachaças de acordo com as variáveis investigadas, nem de interação entre essas variáveis. Quanto ao $\mathrm{Pb}$, foi encontrada interação entre as variáveis teor alcoólico e procedência, com modificação dos efeitos principais. Nos copos de Cachoeira do Brumado, a cachaça com menor teor alcoólico apresentou um teor médio de chumbo 0,01 $\mathrm{mg} / \mathrm{L}$ superior à cachaça de maior graduação alcoólica. As amostras de cachaça com menor teor alcoólico mantidas em contato com os copos oriundos de Cachoeira do Brumado apresentavam um teor médio de chumbo $0,01 \mathrm{mg} / \mathrm{L}$ maior do que as amostras de mesmo teor alcoólico armazenadas nos copos provenientes de Santa Rita (Tabela 5).

Embora o pH das cachaças não tenha sido determinado, é conhecido que este pode influenciar a corrosão da pedra-sabão, com o menor valor associado à maior erosão da pedra-sabão, contribuindo para maior transferência de metais do material para o alimento ou líquido que esteja em contato. ${ }^{2}$ No presente estudo, que se mostra pioneiro em avaliar a transferência de elementos da pedra-sabão para bebidas alcoólicas, foi notado que o $\mathrm{Pb}$ apresentou maior nível no ensaio com cachaça com graduação alcoólica de $40 \%$, indicando que o teor alcoólico apresenta influencia na migração do metal, sendo que a cachaça com teor alcoólico igual a $46 \%$ apresentou menor efeito sobre a migração de $\mathrm{Pb}$, independente da procedência do esteatito. Apesar do $\mathrm{Cu}$ também apresentar esse comportamento, o mesmo é atribuído à adsorção e não a um possível efeito protetor da maior graduação alcoólica da bebida (46\%).

Adicionalmente, em estudo com simulante de alimento à base de ácido acético a 3 e a 5\%, a pedra-sabão in natura foi considerada imprópria para o contato com alimentos em função da transferência de quantidades nocivas de $\mathrm{Ni}$, sendo recomendada a cura dos recipientes antes do primeiro uso. ${ }^{9}$ No entanto, no presente estudo o comportamento da pedra-sabão não-curada em relação à transferência de contaminantes inorgânicos para cachaças se mostra aceitável, inclusive com possibilidade de redução na quantidade de $\mathrm{Cu}$ presente em função do tempo de contato.

\section{CONCLUSÃO}

A maior parte das cachaças produzidas no Quadrilátero Ferrífero de Minas Gerais avaliadas neste estudo apresentou conformidade à legislação brasileira no que tange aos teores dos contaminantes As, $\mathrm{Cd}, \mathrm{Cu}, \mathrm{Ni}$ e $\mathrm{Pb}$. Os ensaios de migração evidenciaram que o teor alcoólico da cachaça pode influenciar na transferência de contaminantes inorgânicos dos copos de pedra-sabão, determinando os teores dos elementos transferidos, porém sem extrapolar os limites de contaminantes previsto na legislação brasileira vigente. Os resultados apresentam indícios de que o armazenamento de cachaças em recipientes de esteatito in natura pode ser uma alternativa para reduzir o teor de $\mathrm{Cu}$ em cachaças, colaborando para que o teor final do elemento esteja em consonância com os limites legais de consumo da bebida.

\section{AGRADECIMENTOS}

Os autores agradecem à A. P. F Oliveira, à R. F. Milani e ao professor A. F. Pereira pela ajuda nas análises e interpretação dos resultados, bem como à Fundação de Amparo à Pesquisa do Estado de Minas Gerais (FAPEMIG) pelo apoio financeiro (APQ-01007-10).

\section{REFERÊNCIAS E NOTAS}

1. Storemyr, P.; Mater. Charact. 2004, 53, 191.

2. Quintaes, K. D.; Cerâmica 2006, 52, 298.

3. Magee, P.; Barber, D.; Sobur, M.; Jasim, S.; Arab. Arch. Epig. 2005, 16, 129.

4. Namdar, D.; Stacey, R. J.; Simpson, S. J.; J. Archaeol. Sci. 2009, 36, 2507.
5. Jones, R. E.; Kilikoglou, V.; Olive, V.; Bassiakos, Y.; Ellam, R.; Bray, I. S. J.; Sanderson, D. C. W.; J. Archaeol. Sci. 2007, 34, 626.

6. Santi, P.; Renzulli, A.; Antonelli, F.; Alberti, A.; J. Archaeol. Sci. 2009, 36, 2493.

7. Jordt-Evangelista, H.; Silva, M. E.; REM: Rev. Esc. Minas 2005, 58, 11.

8. Farias, R. F.; J. Serb. Chem. Soc. 2005, 70, 261.

9. Quintaes, K. D.; Farfan, J. A.; Morgano, M. A.; Mantovani, D. M. B.; Food Addit. Contam. 2002, 19, 134.

10. Warscheid, T. H.; Braams, J.; Int. Biodeter. Biodegr. 2000, 46, 343.

11. Viles, H.; Geomorphology 1995, 13, 21.

12. Quintaes, K. D.; Farfan, J. A.; Tomazini, F. M.; Morgano, M. A.; Arch. Latinoam. Nutr. 2006, 56, 275.

13. Heikkinen, P. M.; Räisänen, M. L.; Appl. Geochem. 2009, 24, 1224.

14. Quintaes, K. D.; Farfan, J. A.; Morgano, M. A.; Haj-Isa, N. M. A.; Grasas y Aceites 2007, 58, 334.

15. Quintaes, K. D.; Farfan, J. A.; Morgano, M. A.; Tomazini, F. M.; Mantovani, D. M. B.; Cienc. Tecnol. Aliment. 2004, 24, 397.

16. Maduabuchi, J. M. U.; Nzegwu, C. N.; Adigba, E. O.; Oragwu, C. I.; Agbo, F. N.; Agbata, C. A.; Ani, G. C.; Orisakwe, O. E.; Journal of Health Science 2008, 54, 335.

17. Brasil, Ministério da Agricultura, Pecuária e Abastecimento, Instrução Normativa No 13 de 29/06/2005. Diário Oficial da União - Seção 1, N 124 de 30 de Junho de 2005; p. 3-4.

18. Caldas, N. M.; Raposo, J. J. L.; Gomes Neto, J. A.; Barbosa, J. F.; Food Chem. 2009, 113, 1266.

19. http://www.abrabe.org.br/cachaca.php , acessada em Fevereiro 2010.

20. Nascimento, R. F.; Bezerra, C. W. B.; Furuya, S. M. B.; Schultz, M. S.; Polastro, L. R.; Lima Neto, B. S.; Franco, D. W.; J. Food Comp. Anal. 1999, 12, 17.

21. Fernandes, A. P.; Santos, M. C.; Lemos, S. G.; Ferreira, M. M. C.; Nogueira, A. R. A.; Nóbrega, J. A.; Spectrochim. Acta B. 2005, 60, 717.

22. Ibanez, J. G.; Carreon-Alvarez, A.; Barcena-Soto, M.; Casillas, N.; J. Food Comp. Anal. 2008, 21, 672.

23. O QFMG engloba 34 cidades de MG, a saber: Barão de Cocais; Belo Horizonte; Belo Vale; Betim; Brumadinho; Caeté; Catas Altas; Congonhas; Conselheiro Lafaiete; Ibirité; Igarapé; Itabira; Itabirito; Itatiaiuçu; Itaúna; Jeceaba; João Monlevade; Mariana; Mário Campos; Mateus Leme; Moeda; Nova Lima; Ouro Branco; Ouro Preto; Raposos; Rio Acima; Rio Manso; Rio Piracicaba; Sabará; Santa Bárbara; Santa Luzia; São Gonçalo do Rio Abaixo; São Joaquim de Bicas; Sarzedo.

24. Borba, R. P.; Figueiredo, B. R.; Cavalcanti, J. A.; REM: Rev. Esc. Minas. 2004, 57, 45.

25. Brasil. Ministério da Saúde. Agência Nacional de Vigilância Sanitária. Decreto $\mathrm{n}^{\circ}$ 55.871, de 26 de março de 1965. Diário Oficial da União; Poder Executivo, de 09 de abril de 1965.

26. INMETRO. Instituto Nacional de Metrologia, Normalização e Qualidade Industrial. DOQ-CGCRE-008. Revisão: 04, 2011.

27. Stata Corporation. Release 11.0. College Station, TX: Stata Corporation, 2010.

28. Fernandes, J. W.; Cardoso, M. G.; Vilela, F. J.; Morais, A. R.; Silva, V. F.; Nelson, D. L.; J. Food Comp. Anal. 2007, 20, 257.

29. ANVISA. Agência Nacional de Vigilância Sanitária. Resolução RDC $n^{\circ} 20$, de 22 de março de 2007. D.O.U. - Diário Oficial da União; Poder Executivo, de 26 de março de 2007.

30. Lima, A. J. B.; Dissertação de Mestrado, Universidade Federal de Lavras, Brasil, 2005.

31. Lima, A. J. B.; Cardoso, M. G.; Guimarães, L. G. L.; Lima, J. M.; Nelson, D. L.; Quim. Nova 2009, 32, 845.

32. Proti, R. S. C.; Dissertação de Mestrado, Universidade Federal de Ouro Preto, Brasil, 2010.

33. Bezerra, O. M. P. A.; Tese de Doutorado, Universidade Federal de Minas Gerais, Brasil, 2002. 